\title{
Benefits of short-term training with vibrotactile biofeedback of trunk sway on balance control in multiple sclerosis
}

\author{
Rust HM${ }^{1}$, Lutz $\mathrm{N}^{2}$, Zumbrunnen $\mathrm{V}^{3}$, Imhof $\mathrm{M}^{3}$, Yaldizli Ö$^{1}$, Haller $\mathrm{V}^{1}$ and Allum JHJ,4* \\ ${ }^{1}$ Department of Neurology, University of Basel Hospital, Basel, Switzerland \\ ${ }^{2}$ Department of Health Professions, Bern University of Applied Sciences, Switzerland \\ ${ }^{3}$ Health Sciences Teaching Centre Basel, Switzerland \\ ${ }^{4}$ Department of ORL, University of Basel Hospital, CH-4031 Basel, Switzerland
}

\begin{abstract}
Background and aims: Patients with multiple sclerosis (MS) suffer from diminished balance control. We examined whether 4 sessions of training with vibrotactile biofeedback (VTfb) of trunk sway could improve their balance control and provide a carry-over effect.

Methods: Baseline trunk sway was first measured for $15 \mathrm{MS}$ patients. Then they received head mounted VTfb of trunk sway which was directionally active when trunk sway exceeded limits set using the baseline assessments. Stance and gait tasks were trained 2 times weekly for 2 weeks with VTfb. Assessments with VTfb were performed at the end of each week. Two weeks later balance was assessed without VTfb to determine if a carry-over effect was present.

Results: Assessments with VTfb showed a significant decrease in trunk sway after 1 and 2 weeks of VTfb training (p<0.02). Carry-over improvements were also present $(\mathrm{p}<0.02)$. The greatest effects were found for tests of standing eyes closed stance on foam which resulted in a $59 \%$ decreased pitch sway angle ( $\mathrm{p}=0.002)$ with $\mathrm{VTfb}$ and a $51 \%$ reduction $(\mathrm{p}=0.03)$ carry-over effect.

Conclusions: This study indicates that balance control in MS patients improves rapidly after one week of training with VTfb and more slowly subsequently. The carry-over effect lasted at least 2 weeks. Future studies should determine, with more weeks of VTfb training, the time course of the slower balance and carry-over improvements following the first rapid improvement in balance control. We conclude that training with VTfb of trunk sway significantly improves balance control in MS patients, and could possibly reduce falls.
\end{abstract}

\section{Introduction}

Multiple sclerosis (MS) is a chronic inflammatory demyelinating disease of the central nervous system (CNS). It is the most common non-traumatic neurological disease in young adults leading to disability with a median prevalence of 83 per 100,000 in Europe [1]. Balance deficits are very common in MS patients [2] and associated with an increased risk of falls with subsequent injuries $[3,4]$. These deficits arise mostly from a combination of various neurological deficits because the clinical presentation and disease course of MS is individually highly variable $[5,6]$.

Balance deficits in MS patients are related to motor symptoms, mainly paresis and spasticity in the lower extremities, decreased somato-sensory proprioception due to slowed sensory conduction, cerebellar disturbances resulting in cerebellar ataxia as well as visual impairment due to decreased vision or oculomotor disorders and fatigue $[5,7,8]$. In addition, cognitive impairment contributes to imbalance in MS patients [9-12]. Of the above-mentioned contributing factors to balance impairment in MS, slowed afferent proprioceptive conduction due to affection of the spinal cord as well as disturbed central integration seem to have pivotal roles [7,8,12-15]. Regarding findings from MRI studies which showed white matter and grey matter affections in early stages of the disease [16,17], it can be assumed on the basis of current concepts of widespread central vestibular networks $[18,19]$, that a disruption of these networks by diffuse white as well as grey matter affection may contribute, in a yet under-recognized amount, to balance abnormalities even in early stages of the disease $[15,16]$. Findings of balance abnormalities in previous studies for which MS patients were assessed shortly after first diagnosis was made and only mild or no focal neurological deficits were reported, supports this assumption $[14,15,20]$.

Postural control in patients with MS has been studied extensively over the last decades [21]. For an overview of the currently available technologies to assess balance in MS see Shanahan et al. [22]. Findings from previous studies assessing body sway in patients with MS [23] could be confirmed when studying balance in MS patients with body worn gyroscopes. In these latter studies, MS patients with clinically apparent minimal disability showed altered body sway compared to healthy controls [20,24-26].

${ }^{*}$ Correspondence to: John Allum, Department of ORL, University of Basel Hospital, CH-4031 Basel, Switzerland, E-mail: john.allum@usb.ch

Key words: relapsing-remitting MS, stance and gait, balance control, trunk sway, vibro-tactile feedback, sensory augmentation

Received: July 02, 2020; Accepted: July 13, 2020; Published: July 23, 2020 
Recently, various rehabilitation techniques have been used to improve balance in MS patients. These include using force plates to display excessive body lean [27], robot assisted gait [28], and virtual reality environments [29]. Another possible approach, often used for peripheral vestibular loss subjects, would be to use vibro-tactile feedback (VTfb) of trunk sway [30,31]. The advantage of the VTfb technique is that the ensuing skin sensations, specifically vibration, are transmitted to the brain over afferent fibres [32]. Such sensory augmentation was first used in patients to improve the balance control of those patients with vestibular loss [33]. However, the original use was to increase military pilot awareness of orientation when flying without vision [34]. Typically, during stance, trunk angle feedback is used, and during gait trunk angular velocity feedback is used, with the instruction to the test subject being to move away from the direction of the vibration when the directional specific vibrator is switched on because its pre-set threshold has been exceeded [33,35]. Kentala et al. [36] were the first to demonstrate that VTfb reduced sway of vestibular loss subjects standing eyes closed. Subsequently this action was shown to be due to vestibular loss subjects learning to reduce coactivation levels in trunk and leg muscles to levels of healthy controls [37].

The vibro-tactile sensory augmentation technique has been used with a variety of persons, apart from those with vestibular loss. These have included healthy young and older adults in addition to patients with diseases: stroke, diabetic neuropathy, and Parkinson's disease (for a review see Ma et al. [38]). To date, according to our knowledge, this technique has not been applied to MS patients, apart from in our recent study [39], despite the known balance disorders of MS patients, as described above. Most of the studies with other patient groups have assumed that the VTfb device should be worn continuously, thereby not depending on possible learning and adaptation effects present as carry-over effects. In this respect it is important to note that VTfb could increase the attention load as discovered with the elderly for cognition tasks, but not for motor tasks, and lead to no balance improvement being observed [40]. For this reason, we have also concentrated on also documenting the carry-over effect of balance training with VTfb of trunk sway in this study.

The VTfb rehabilitation approach as used in this study for improving balance in patients with MS is based on previous experimental work and hypotheses regarding slowed conduction and central integration of proprioceptive sensory signals in patients with MS [7]. In order to augment proprioceptive sensation of trunk sway, we applied VTfb to the skin of the head when sway trunk measured at the centre of mass exceeded a preset threshold. This information was applied directionally. Thereby we aimed to create a rapid artificial "proprioceptive" pathway as a supplementary pathway to that of patients' slowed afferent pathways.

Our previous study had shown that 2 sessions of VTfb training and assessments over 1 week led to trunk sway reductions during stance and gait tasks compared to training without VTfb [39]. The aim of the current study, with a different group of MS patients, was to determine if 2 weeks of VTfb training and assessment twice a week (4 sessions) would yield a more marked improvement of balance control with VTfb than 1 week of VTfb training and assessments as employed by van der Logt et al. [39]. In addition, we wished to determine if a carry-over effect was still present 1 week after training had ceased. This latter aspect was not explored by van der Logt et al. [39]. Thus, the training and assessment procedure we describe below and in figure 1 was used to attempt to provide an initial answer to the question whether the carry-over effect of 4 sessions of training and assessments with VTfb is so strong that continuous wear of the VTfb system for improved balance control might not be necessary. Rather, patients would only need to re-train for a number of weeks with VTfb once the carry-over effect had worn off. The advantage of the technique used in this study is the simplicity of the feedback, which is directly related to movements of the body's centre of gravity, and the placement of the VTfb at the head, which we assumed acted to produce a rapid sensory augmentation for the information received by the patient's proprioceptive and peripheral vestibular systems.

\section{Methods}

\section{Participants}

The protocol of this study was approved by the local ethics committee (Approval 2014-026 Ethical Committee NW Switzerland) and all participants provided prior written informed consent to participate before enrolment in the study. Sixteen participants were recruited from the neurological outpatient clinic of the University of Basel Hospital, Switzerland. One participant discontinued during the study because of hip tendon inflammation (Table 1). Of the remaining 15 patients, 3 were male, 14 had relapsing-remitting MS, and 1 secondary-progressive MS. The mean patient age was $46.9 \pm 12.1$ years, the mean EDSS score was 3.1 [median 3.0, range $1.5-4.0$ ], and mean disease duration was $9.8 \pm 7.4$ years. Inclusion criteria were the diagnosis of MS according to the McDonald criteria [41], no relapse during the last 6 months, subjectively reported restrictions to balance and quantified deficits in balance control noted in the first assessment (for details, see below). Participants were excluded if they were not able to walk 8 meters without walking aids, had other conditions than MS that might affect balance (e.g. vestibular impairment, orthopaedic disorders, and severe comorbidities) or had noticeable cognitive or psychiatric disturbances that might have or proved to have affected testing. Table 1 describes the patients' neurological characteristics.

Participant sensed restrictions in balance control during daily life were estimated using the Dizziness Handicap Inventory (DHI) [42] and the MS Walking Scale (MSWS-12) [43] questionnaires. The DHI consists of 25 questions designed to quantify the subjectively experienced balance deficit. It has satisfactory reliability and validity values when applied to MS patients [44]. The higher the DHI score the greater is the subjectively experienced disability. MSWS-12 is a validated subjective measure of walking ability of MS patients [43].

Balance control was measured with a SwayStar ${ }^{\mathrm{TM}}$ system (Balance International Innovations $\mathrm{GmbH}$, Iseltwald, Switzerland) which contains two fibre-optic gyroscopes mounted on a converted motorcycle kidney belt. These were aligned to measure lower trunk pitch (posterior-anterior sway) and roll (medial-lateral sway) angular velocities. The velocities were sampled at $100 \mathrm{~Hz}$ and then transferred to a computer using Bluetooth ${ }^{\mathrm{TM}}$ communication. Pitch and roll angles are computed on-line using trapezoid integration of the velocity samples. Prior to participating in the experiments, subjects underwent a comprehensive clinical neurological examination with screening for inclusion and exclusion criteria. Table 1 describes the participants' neurological deficits resulting from these examinations.

\section{Intervention}

Vibrotactile feedback (VTfb) of trunk sway was provided to study participants using an add-on device of Swaystar called Balance Freedom ${ }^{\mathrm{TM}}$. This feedback system consists of 8 vibrators positioned at 45-degree intervals around a head band. The vibrators were standard mobile telephone vibrators operating at $150 \mathrm{~Hz}$ and switched on 
Table 1. Patient neurological characteristics

\begin{tabular}{|c|c|c|c|c|c|c|c|}
\hline Subj & MS type & gender & $\begin{array}{l}\text { Disease duration, } \\
\text { years }\end{array}$ & EDSS & $\begin{array}{l}\text { Clinically apparent focal neurological deficits contributing to } \\
\text { balance disorder }\end{array}$ & DHI Score & MSWS Score \\
\hline 1 & RRMS & $\mathrm{f}$ & 13.1 & 2.5 & Paresis LRE M5-/5 & 60 & 29 \\
\hline 2 & RRMS & $\mathrm{f}$ & 4 & 3.5 & Paraparesis, LLE M5-/5 & 38 & 33 \\
\hline 3 & RRMS & $\mathrm{f}$ & 7.9 & 4.0 & $\begin{array}{l}\text { Paraparesis, hip flexion both sides M4+/5, knee flexion LRE } \\
\text { M4+/5 }\end{array}$ & 30 & 37 \\
\hline 4 & RRMS & $\mathrm{f}$ & 2.3 & 2.5 & Slight proprioceptive deficit LRE, motor fatigue & 24 & 25 \\
\hline 5 & RRMS & f & 6.1 & 4.0 & Hypaesthesia in both upper and lower extremities, motor fatigue & 40 & 36 \\
\hline 6 & RRMS & $\mathrm{f}$ & 11.1 & 3.5 & Proximal paresis LLE M5-/5 with proximal increased tone & 48 & 35 \\
\hline 7 & SPMS & $\mathrm{m}$ & 11.3 & 3.5 & Paraparesis, hip flexion M5-/5 paraspasticity left $>$ right & 62 & 45 \\
\hline $8 *$ & RRMS & $\mathrm{f}$ & 15.7 & 4.5 & $\begin{array}{l}\text { Paraparesis, hip flexion, knee flexion and knee extension } \mathrm{M} 4+/ 5 \\
\text { BLE, increased tone BLE right }>\text { left, severe proprioceptive } \\
\text { deficit BLE }\end{array}$ & 48 & 52 \\
\hline 9 & RRMS & $\mathrm{f}$ & 27.4 & 3.0 & $\begin{array}{l}\text { Paraparesis, hip flexion and knee flexion M5-/5, increased tone } \\
\text { BLE right }>\text { left }\end{array}$ & 34 & 42 \\
\hline 10 & RRMS & $\mathrm{f}$ & 20.8 & 3.5 & Paraparesis, hipflexion M 4-/5 LLE & 12 & 30 \\
\hline 11 & RRMS & $\mathrm{m}$ & 1.7 & 3.5 & Paraparesis, hip flexion M 4-/5 LLE only, paraspasticity & 18 & 23 \\
\hline 12 & RRMS & $\mathrm{f}$ & 2.1 & 2.5 & Paraparesis, hip-flexion M 4-/5 LRE & 12 & 25 \\
\hline 13 & RRMS & $\mathrm{f}$ & 11.1 & 3.0 & Paraparesis, hip flexion M4/5 and dorsiflexion M4/5 LLE & 14 & 21 \\
\hline 14 & RRMS & $\mathrm{m}$ & 13.5 & 3.0 & $\begin{array}{l}\text { Paraparesis right }>\text { left M5-/5, right sided hemispasticity } \\
\text { pronouned in RLE }\end{array}$ & 64 & 153 \\
\hline 15 & RRMS & $\mathrm{f}$ & 6.8 & 3.5 & Paresis LLE, knee flexion M4/5, increased tone LLE & 90 & 32 \\
\hline 16 & RRMS & $\mathrm{f}$ & 1.1 & 3.0 & $\begin{array}{l}\text { Paraparesis M5-/5, right > left, Hypaesthesia lower extremities, } \\
\text { afferent deficit }\end{array}$ & 2 & 15 \\
\hline
\end{tabular}

MS: Multiple Sclerosis; RRMS: Relapsing Remitting Multiple Sclerosis; SPMS: Secondary Progressive Multiple Sclerosis; EDSS: Expanded Disability Status Scale; LRE: Lower Right Extremity; LLE: Lower Left Extremity; BLE: Both Lower Extremities; DHI: Baseline Dizziness Handicap Inventory Score; MSWS Baseline MS Walking Score. $8 *$ means the patient was excluded due to hip tendon inflation acquired during the study.

when a sway threshold was exceeded in the direction of the vibrator. For example, if a sway threshold for forward pitch was exceeded, the vibrator in the middle of the forehead came on. Likewise, for backward movement exceeding threshold, the vibrator at the back of the head came on. Left and right sway supra-threshold sway caused the vibrators over the ears to be switched on. For sway in the diagonal directions, forward and left, for example, the vibrator between the forehead and left ear vibrators was switched on when its threshold was exceeded. Thus, when the head was aligned in the head straight-ahead position, the vibro-tactile feedback (VTfb) provided by the vibrators was aligned with the axes of the gyroscopes. The controller for the vibrators was connected directly with SwayStar ${ }^{\mathrm{TM}}$. Task-specific thresholds for trunk sway angles (used during stance and tandem steps tasks) and angular velocities (used during gait tasks) were set in the controller based on pitch and roll measures obtained from the first (baseline) assessment and readjusted based on measures from the third assessment (the first assessment after baseline without VTfb (Figures 1 and 2). The threshold amplitudes were calculated as $40 \%$ of the $90 \%$ ranges of pitch and roll sway angle or angular velocity. Thus, the threshold ranges were set at $80 \%$ ( $40 \%$ for each side) of the $90 \%$ ranges, i.e. at the $72 \%$ range. To determine the $90 \%$ ranges, the total peak-to-peak range of each variable was determined over the trial duration for a task and this range was split into 40 bins. Samples were then sorted into the appropriate bins to build a histogram of the samples. The range from the lower $5 \%$ and the upper $95 \%$ of the histogram was used to define the $90 \%$ range. The thresholds were individually set for each task. More detailed information on the threshold settings can be found in van der Logt et al. [39].

\section{Procedure}

Over a period of 4 weeks, all participants performed a series of assessment and training sequences of gait and stance tasks. The order of assessments and training sessions is presented in figure 1. Basically, the study procedure consisted of three phases: An intervention phase (weeks
1 and 2 providing training and assessment with VTfb of trunk sway), a phase of 1 week with no intervention (week 3 ) and an assessment of the carry-over effects at the beginning of week 4 . Assessments without VTfb were always carried out on Mondays of weeks 1, 2 and 4. In weeks 1 and 2 the assessments without VTfb were used to set and reset the VTfb thresholds, respectively. In weeks 2 and 4, the assessments on Mondays were also used to assess the carry-over effect of VTfb training. Training with VTfb was performed on Wednesdays during weeks 1 and 2. Training with VTfb was also provided before the assessments on Fridays of weeks 1 and 2 (Figure 1). For weeks 1 and 2 the assessments on Fridays provided information on whether on-line VTfb after training with VTfb aided balance control. A break of 10 minutes was provided between training and assessment sessions on Fridays to avoid fatigue. Thus, assessments 1 and 3 in weeks 1 and 2 were performed with no VTfb and assessments 2 and 4 in the same weeks with VTfb. The last assessment, 5, in week 4 was without VTfb (Figure 1).

\section{Control procedure}

In a previous study [39] we demonstrated that training with VTfb over 1 week brought a consistently greater improvement in trunk sway of MS patients during balance tasks compared to training without VTfb and the immediate carry-over effect was also greater. This previous study was designed to be a control study for the current and future studies. The previous study first had a balance assessment without $\mathrm{VTfb}$, then for one group of MS patients, 2 sessions of training and assessment with VTfb to determine the feedback effect followed by an assessment without VTfb to determine the immediate carry-over effect. Another comparison group of MS patients received all assessments and training without VTfb. That is the number of training/assessments sessions per week was the same as in the current study. However, in the current study there were 2 of weeks of VTfb training and assessments rather than 1 week, and the carry-over effect was examined after a pause of 1 week. 


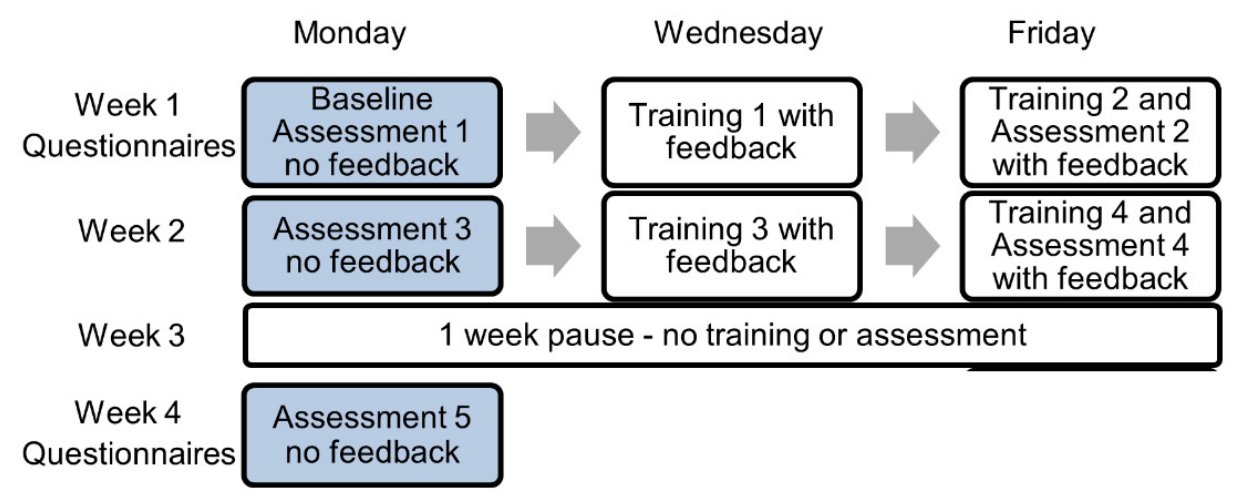

Figure 1. Schema of the timing of balance assessments and training sessions, with and without, vibro-tactile balance feedback of trunk sway. The subjects were seen 3 times a week over 2 weeks and after one week (week 3) having neither assessments nor training. A final assessment (5) took place on Monday of week 4

\section{Effect of trunk pitch sway feedback for: Standing on 2 legs, eyes closed on foam}

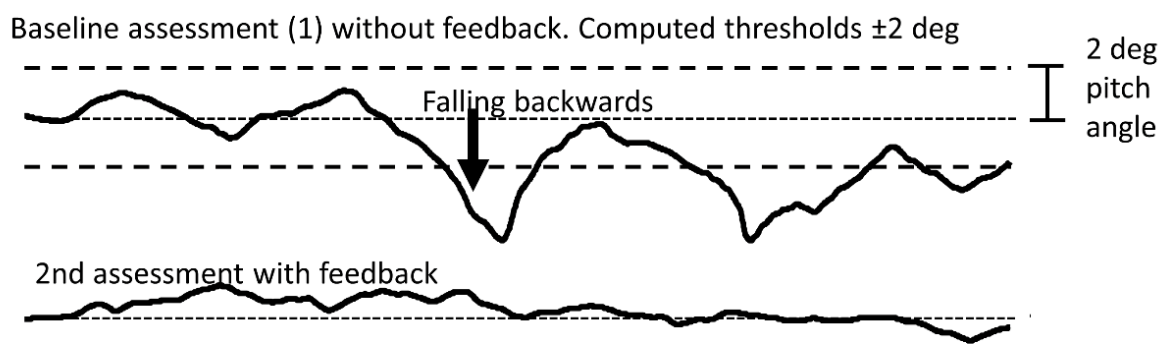

3rd assessment without feedback. Computed thresholds $\pm 0.5 \mathrm{deg}$

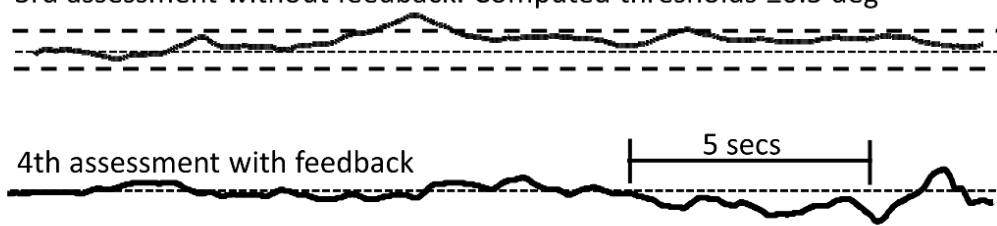

Figure 2. Example of the reduction in vibro-tactile feedback thresholds as the subject improves balance control. The uppermost traces show the trunk pitch sway angle for the first, baseline, assessment of the task standing on 2 legs, eyes closed, on foam support. The dashed lines show the computed feedback thresholds used for the training and second assessment testing with feedback. The pitch sway angle for this assessment is shown in the second trace. The third assessment without feedback shows the recomputed reduced feedback thresholds as dashed lines. The trunk pitch sway for the fourth assessment is shown in lowermost trace

\section{Assessment and training tasks}

Eleven tasks were used for the assessments and 7 were selected for training sessions (Table 2). Thus, 4 tasks were not trained in order to determine if these tasks improved as part of a generalized improvement in balance. Selection of tasks was based on previous studies, which indicated the ability to determine, with these tasks, the presence of subclinical balance disorders in MS patients [20,25]. Assessments and training were always performed without shoes to avoid measurement variations due to shoe types. To avoid diurnal differences in fatigue levels affecting the results, assessments and training were always performed at the same preferred time of the day for each patient.

For two-legged stance tasks, participants were asked to stand still with eyes open or closed on a firm or foam surface in a normal, comfortable standing position, lateral borders of the feet hip width apart, and arms hanging alongside the trunk. The foam surface was
Table 2. Stance and gait tasks for assessment and training sessions. The order in which the tasks were performed is as listed. As indicated, some tasks were only used for assessment not for training

\begin{tabular}{|l|c|}
\hline \multicolumn{1}{|c|}{ Task } & Training/Assess \\
\hline Standing on 2 legs, eyes closed, 20 seconds & Both \\
\hline Standing on 1 leg, eyes open, 20 sec & Not trained \\
\hline Standing on 2 legs, eyes open, on foam, 20 seconds & Not trained \\
\hline Standing on 2 legs, eyes closed, on foam, 20 seconds & Both \\
\hline Standing on 1 leg, eyes open, on foam, 20 seconds & Both \\
\hline Tandem stance, eyes open, 30 seconds & Both \\
\hline 8 tandem steps, eyes open & Both \\
\hline Walking 3 meters while pitching the head up and down & Not trained \\
\hline Walking 3 meters, eyes closed & Both \\
\hline Walking over 4 barriers & Not trained \\
\hline Walking 8 meters, eyes open & Both \\
\hline
\end{tabular}


$10 \mathrm{~cm}$ high, $44 \mathrm{~cm}$ width and $204 \mathrm{~cm}$ long and had a density of 25 $\mathrm{kg} / \mathrm{m}^{3}$. For eyes open stance tasks, patients were asked to focus on a point at eye height 5 meters away. During one-legged tasks, patients chose their better leg to stand on. Stabilizing their raised leg against the standing leg was not allowed. All walking tasks were measured over 3 meters, except walking 8 meters and walking over four low barriers. The barriers were $24 \mathrm{~cm}$ in height and spaced one meter apart. During all tasks patients were asked not to talk, and a spotter stood next to them to aid in case of a loss of balance control. For the assessment sequences, each task was performed once and in training sequences, each task was repeated five times. Tasks were stopped if the patient lost balance or completed the task. During the training and the assessment sequences, patients were requested to reduce sway as much as possible. When the patients received VTfb of trunk sway, they were requested to try to avoid activating the vibrotactile transducers or, if the VTfb was activated, to move away from the direction indicated by the feedback.

\section{Outcome measures}

For each task of the assessments, peak-to-peak and $90 \%$ ranges for pitch velocity (pv), pitch angle (pa), roll velocity (rv) and roll angle ( $\mathrm{ra}$ ) and duration (dur) were measured. Both angles and angular velocities were measures as the cone of body stability depends on both. If the patient lost control of balance during a gait task, that trial's duration was not entered in the analysis to avoid misrepresenting this duration as a fast task completion. For stance tasks the duration was reduced to the time-point when the subject lost control of balance. We concentrated on one primary measure, a global balance control index $(\mathrm{BCI})$ provided by the SwayStar software to compare balance control between assessments. The BCI is a single combined score from several tasks [45]. For each subject, the BCI computed by the software is compared to age matched healthy values of persons with an age \pm 5 years of the test subject. For the mean normal value displayed in figure 4 this involved a comparison with values of 50 healthy normal subjects of the same mean age \pm 5 years as the MS patients of this study (mean age 47 years). The index combines peak-to-peak measures from several tasks into one value. Thus, the BCI is an additive composite score based on measures from several tasks: From the task standing on 2 legs on foam with eyes closed $\left(2{ }^{\star} \mathrm{pv}\right)$, for walking 8 tandem steps $\left(1^{\star}\right.$ ra), for walking $3 \mathrm{~m}$ eyes closed $\left(1.5^{\star} \mathrm{pv}+20^{\star}\right.$ dur $)$, walking $3 \mathrm{~m}$ while pitching the head up and down $\left(1.5^{\star} \mathrm{pv}\right)$. That is

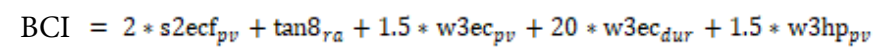

The basis for this selection of measures and coefficients is a stepwise discriminant analysis described in Allum and Adkin [46]. This combination of the selected balance outcome measures has been shown previously to have a high accuracy in detecting patients with impaired balance [46]. As this index revealed differences for assessments with $\mathrm{VTfb}$, we examined as secondary measures $90 \%$ ranges of all trunk sway measures and task durations.

Subjective balance impairment was measured using the above mentioned DHI and MSWS-12 questionnaires at the beginning of weeks 1 and 4 (Figure 1) in order to capture subjective carry-over effects.

\section{Statistics}

One-way analysis of variance with repeated measures was performed to compare baseline assessment 1 results with those of assessments 2 through 5 after checking that measures were normally distributed. BCI values, mean duration, and the mean of the $90 \%$ ranges for $\mathrm{pv}, \mathrm{pa}, \mathrm{rv}$, and ra for balance tasks, were compared between assessments after checking that the data was normally distributed. Paired Students t-Tests with Bonferroni corrections were performed for 3 comparisons of post-hoc analyses, those of assessments 1 with 2 and 4 (assessments with VTfb) and assessment 1 with 3 and 5 (no VTfb). The level of alpha was set at $5 \%$.

\section{Results}

The improvements we observed after providing vibrotactile feedback (VTfb) of trunk sway to MS patients in 5 of the 7 trained stance and gait tasks we assessed is illustrated by the typical patient pattern shown for the task standing eyes closed on foam in Figure 2 and documented for these 5 tasks in Table 3. As described, below the other 2 trained tasks showed faster gait speeds with no decreases in trunk sway velocities. We first describe the effects of VTfb on the most difficult stance, standing eyes closed on foam, then the results for the BCI, finally gait results.

The sway amplitude reductions of a typical subject for the task in Figure 2, standing eyes closed on foam follow the pattern of mean population values shown in Figure 3. There was a significant reduction in sway amplitude as listed in Table 3 for pitch angle during this task. The baseline values for this task as measured during assessment 1 were, on average, greater than the $95 \%$ range of normal age-matched controls (Figure 3). (Normal control data was obtained from Hegeman et al. [45]). Following 1 week of training with VTfb, sway values decreased

Table 3. Significant differences for trunk sway in the form of probability (p) values for paired t-tests between initial and follow-up assessments 2 and 4 , for $90 \%$ ranges. $\mathrm{N}=15$ subjects. A: pitch sway and BCI, B: roll sway.

Significant differences with respect to values for assessment 1 that have t-test probability values less than 0.05 marked with bold text, trends $0.05<\mathrm{p} \leq 0.1$ without bold text. *stands for $\mathrm{p} \leq 0.05$ after Bonferroni correction for 3 comparisons; \# stands for $0.1 \geq p \geq 0.05$ after Bonferroni correction for 3 comparisons; ns, no significant difference (2-sided t test).

The gait task w 8 meo had unchanged gait speed $(1.1 \mathrm{~m} / \mathrm{s})$, whereas the task w $8 \tan$ had a decreased duration by $3.0 \mathrm{~s}$ for assessment $4(\mathrm{p}=0.04)$ compared to assessment 1 .

The tasks listed are all trained tasks.

The Balance Control Index (BCI) is a composite score from based on measures from several tests (s2ecf, w8tan, w3mhp, w3mec) see methods and Allum and Adkin 2003 [46].

Assessments 2 and 4 are with VT feedback of trunk sway after 1, respective 2 weeks of VT training.

\begin{tabular}{|c|c|c|c|c|}
\hline \multirow{2}{*}{\begin{tabular}{c|c|} 
A \\
Assessment
\end{tabular}} & \multicolumn{2}{|c|}{ Pitch Angle (deg) } & \multicolumn{2}{|c|}{ Pitch Velocity (deg/sec) } \\
\hline & 2 & 4 & 2 & 4 \\
\hline \multicolumn{5}{|l|}{ Stance tasks } \\
\hline s2ec & $0.006 *$ & 0.045 & ns & ns \\
\hline s2ecf & $0.016 *$ & $0.0032 *$ & 0.05 & $0.023 \#$ \\
\hline Tandem stance & ns & 0.077 & ns & 0.1 \\
\hline \multicolumn{5}{|l|}{ Gait tasks } \\
\hline w8tan & 0.08 & $0.014 *$ & ns & ns \\
\hline \multirow[t]{2}{*}{ w8meo } & ns & $0.03 \#$ & $0.009^{*}$ & $0.006 *$ \\
\hline & \multicolumn{2}{|c|}{ BCI score } & & \\
\hline BCI & $0.002 *$ & $0.009 *$ & & \\
\hline B & \multicolumn{2}{|c|}{ Roll Angle (deg) } & \multicolumn{2}{|c|}{ Roll Velocity (deg) } \\
\hline Assessment & 2 & 4 & 2 & 4 \\
\hline \multicolumn{5}{|l|}{ Stance tasks } \\
\hline $\mathrm{s} 2 \mathrm{ec}$ & 0.037 & 0.058 & ns & ns \\
\hline s2ecf & ns & 0.098 & ns & $0.028 \#$ \\
\hline Tandem stance & 0.086 & $0.044 \#$ & 0.094 & 0.05 \\
\hline \multicolumn{5}{|l|}{ Gait tasks } \\
\hline w8tan & 0.07 & $0.005^{*}$ & ns & 0.1 \\
\hline w8meo & $0.015^{*}$ & ns & $0.02 \#$ & 0.09 \\
\hline
\end{tabular}

s - standing, 2 - two legs, 1- one leg, eo - eyes open, ec - eyes closed, f - foam support, w -walking, $8 \mathrm{~m}-8$ metres, $8 \tan -8$ tandem steps. 


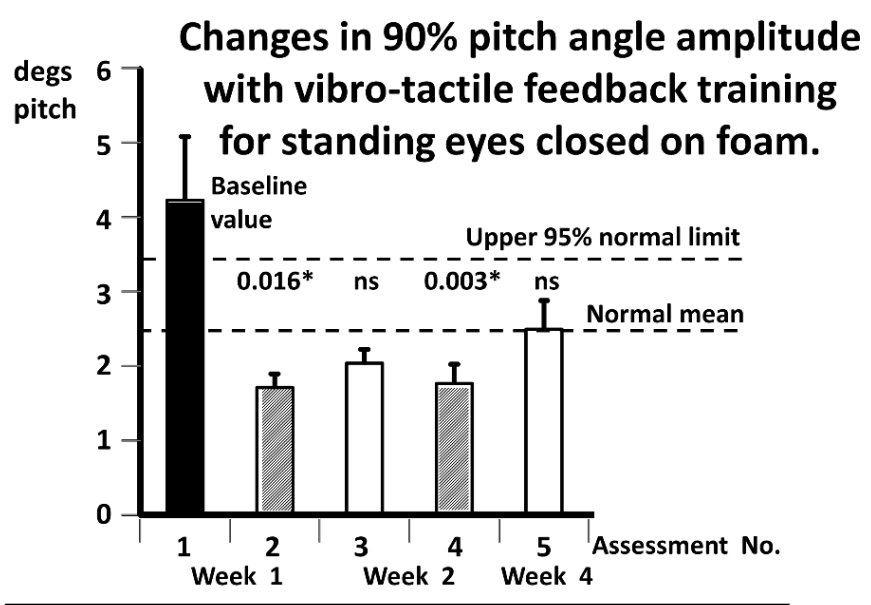

Baseline assessment without VT fb

Assessment with VT fb after training 1 \& 2 weeks with VT fb Assessments without VT fb

Figure 3. Changes in population mean values of the $90 \%$ range of trunk pitch sway angle for the task standing on 2 legs, eyes closed, on foam support during the first and subsequent assessments. The test conditions for each assessment, with and without vibrotactile feedback (VTfb), are explained by the insert below the column diagram. The height of the column represents the mean sample population value for each assessment and the vertical bar on the column, the standard error of the mean. The normal mean and upper $95 \%$ limit values for normal control subjects of the same mean age ( \pm 5 years) as the study MS patients are shown by the dashed lines (data from Hegeman et al. [45]). P values for test comparisons between assessment 1 and the assessment number noted below the column are listed above the columns. Those $\mathrm{p}$ values that are significant after Bonferroni corrections for 2 comparisons ( 1 with 2 and 4; 1 with 3 and 5) are marked with an asterisk

significantly (compare values for assessments 1 and 2 in figures 2 and 3). When tested again at the beginning of the next week (assessment 3), there was a carry-over effect present in the form of reduced sway for this task, which was, however, not significantly less than the baseline values of assessment 1. As shown in Figures 2 and 3, with the reduced thresholds based on measures recorded during assessment 3 , the effect of additional training with VTfb in the second week (as measured by assessment 4) revealed a more significant reduction with respect to the baseline assessment 1 but showed little change from the measures of the previous assessment with VTfb (assessment 2). Again, the subsequent assessment without feedback (assessment 5) revealed a non-significant reduction in sway with respect to assessment 1 . That is, assessment 5 without VTfb revealed a carry-over effect value but with pitch sway greater (but not significantly so) than that of assessment 4, but also not significantly less than the mean of assessment 1.

Figure 4 illustrates that the global balance control index (BCI) comprising several test measures (see methods) followed a similar pattern as described above for pitch angle measures for standing eyes closed on foam (Figure 3). As shown in Table 3 and Figure 5, respectively, the measures pitch velocity for standing eyes closed on foam and the roll angle for tandem gait which feature in the BCI showed significant sway reductions with respect to assessment 1 . In contrast, none of the other gait measures comprising the $\mathrm{BCI}$, for example, pitch velocity for walking while pitching the head up and down, yielded significant changes with respect to assessment 1 . However, the simplest gait task, walking $8 \mathrm{~m}$ eyes open, which does not feature in the BCI yielded significant differences with respect to assessment 1 consistent with the pattern described above: assessment with VTfb after training with VTfb producing the least sway with values close to those of age-matched control subjects (Table 3, Figure 6).
As Goutier et al. [47] noted, increased gait speed leads to increased trunk sway. Therefore, for gait tests we examined whether increases occurred in gait speed in the form of reduced task durations over the fixed trial distances occurred with unchanged or decreased sway amplitudes. One of the 2 gait tasks listed in table 3 with significant reductions in trunk sway, "walking 8 tandem steps" (w8tan) showed reduced task durations $(\mathrm{p}<0.05)$ both with and without VTfb for assessments 4 and 5, respectively. There was no change in gait speed ( $>0.05)$ for the other task listed in Table 3 with reduced sway: "walking $8 \mathrm{~m}$, eyes open" (w8meo). In contrast, the task "walking $3 \mathrm{~m}$, eyes closed" (w3mec) showed no reduction in trunk sway but a reduction

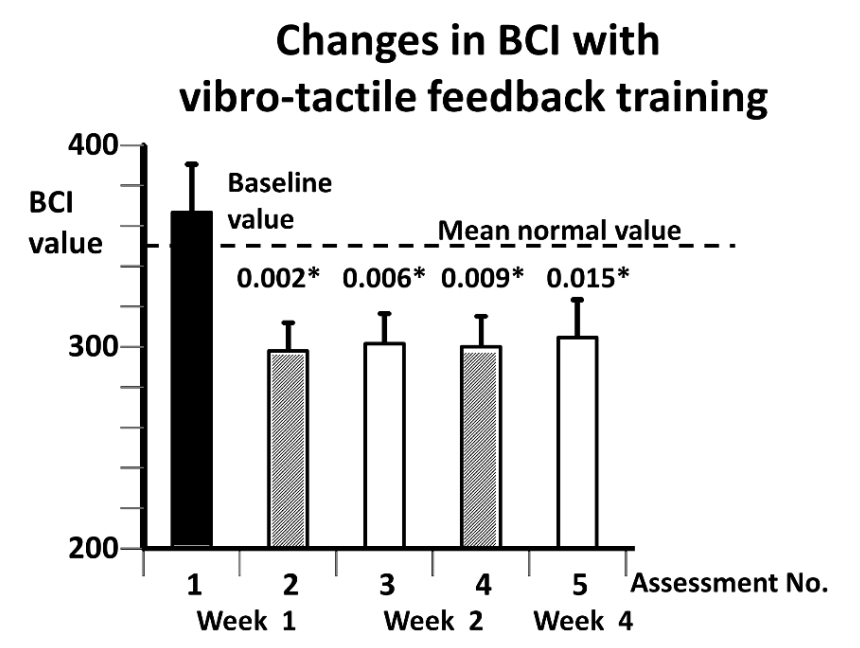

Baseline assessment without VT fb

Assessment with VT fb after training 1 \& 2 weeks with VT fb

Assessments without VT fb

Figure 4. Changes in the population sample population mean Balance control Index (BCI) values for the first in comparison to subsequent assessments. Details of the figure are provided in the legend to figure 3 .

\section{Changes in $90 \%$ roll angle amplitude with vibro-} tactile feedback training for walking 8 tandem steps.

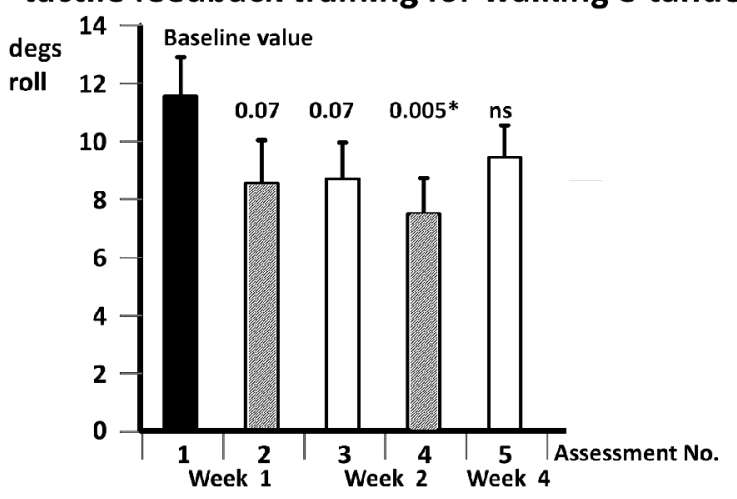

Baseline assessment without VT fb

Assessment with VT fb after training 1 \& 2 weeks with VT fb

Assessments without VT fb

Figure 5. Changes in population mean values of the $90 \%$ range of trunk roll sway angle for the task walking 8 tandem steps during the first and subsequent assessments. Mean values associated with increased gait speed are marked with \#. Other details of the figure are provided in the legend to figure 3 


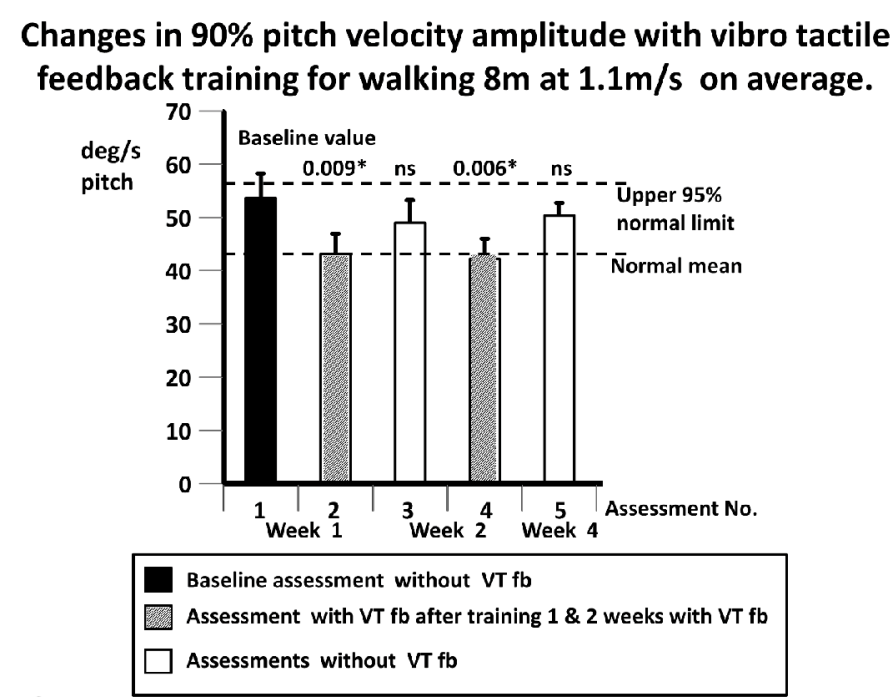

Figure 6. Changes in population mean values of the $90 \%$ range of trunk pitch sway angular velocity for the task walking $8 \mathrm{~m}$ during the first and subsequent assessments. Mean values associated with increased gait speed are marked with \#. Other details of the figure are provided in the legend to figure 3

of mean duration from 5.4 for the baseline assessment 1 to 4.4 secs $(\mathrm{p}=0.01)$ for assessment 4 . This later measure features in the BCI value and therefore contributed to its reduction with VTfb. In summary, all of the trained tasks improved in trunk sway angle and angular velocity amplitudes and/or gait task speed. However, none of the untrained gait tasks showed improvements in gait speed or decreases in trunk sway amplitudes following training with VTfb.

On average, the subjective assessments of walking abilities and the effect of dizziness captured with the MSWS-12 and DHI questionnaires, respectively showed unchanged scores over the 4 week monitoring period; $32 \pm 10$ (mean and standard deviation) compared to $31 \pm 9$ for MSWS-12, and $37 \pm 25$ compared to $34 \pm 26$ for DHI.

\section{Discussion}

The results of this study indicate that providing MS patients VTfb of trunk sway as angle feedback during stance tasks and angular velocity feedback during gait tasks yields significant improvement in balance control with VTfb after 4 training sessions over 2 weeks. The reduction of trunk sway and/or increased gait speed with respect to baseline was greater after 2 weeks, compared to 1 week, as revealed by the increased significance for assessment 4 with respect to baseline (Figures 3-6 and Table 3). However, there was no significant difference after training between the first- and second-weeks' test results, indicating a rapid learning during the first week, and a slightly improved effect during the second week. When subjects were reassessed without feedback 1 week after VTfb training had ended, a carry-over effect was observed which was less significant than the feedback effect. These results raise important questions regarding the number of VTfb training sessions required to achieve the best improvement in balance control, the extent of improvement brought about by VTfb training as a carry-over effect, and the period of time the carry-over effect will last. Future studies should attempt to answer these questions in a more detailed manner than was possible in this study limited to 2 weeks of VTfb training by using a greater number of training and follow-up weeks. Thus the question arises whether it would be possible to quantify the slower rate of improvement after the first week of training with VTfb and the rate of reduction of the carry-over effect over time with longer periods of training and longer follow-up times tracing the carry-over effect. Knowing this information would provide guidelines for the patient's training regime, be it in the clinic or with a take-home device. As the test subjects' subjective assessment of balance capabilities as registered by the DHI and MSWS questionnaire scores did not improve significantly, two further questions arise: Would more weeks of VTfb training improve these subjective assessments? Does the lack of subjective improvement affect subject motivation?

The carry-over effect, relative to the baseline assessment was less significant after an additional week of training with VTfb followed by a pause of one week than before the additional week of training. This raises the question whether the additional week of training or the pause had a detrimental influence on the carry-over effect. This question would need to be answered in future studies by tracking balance changes with a more weeks of training and carry-over effects with more follow-up weeks once training had ceased. Because assessments are also a form of training, the procedure to be used with a control group is complicated as discussed in our earlier study [39]. One could argue that the control group should be trained and assessed with randomly presented VTfb (that is, not driven or partially driven by trunk sway) as other authors have used $[48,49]$. The advantage of such a control procedure is that subjects would wear the headband from which they expect to receive feedback. The disadvantage of such a control procedure is that it could act as distracting dual task, as the study participants must ignor the "false" signal, thereby possibly leading to worse balance control than if no vibro-tactile signal was present as we have used in our control procedure [39].

Most studies to date using VTfb as in this study have demonstrated that one session of training with VTfb yields significantly reduce trunk sway $[31,35,50]$. Our current results concur with this conclusion. In this respect the effect is similar to practicing slipping on a floor [51] or learning to stand upright on a multi-axis balance board [52]. In the latter study, performance was also stable after 2 sessions of training within 1 week and retained for the following week. With a training period of 2 weeks we observed that further reductions in trunk sway amplitudes could be achieved when assessed with VTfb. Given that further improvement with respect to baseline might be observed with more VTfb training, future studies would need to extend the amount of VTfb training in order to show whether after the first week a general slower improvement in balance with VTfb training occurs, and specifically, whether further increases in the significance with respect to baseline also occur for the carry-over effect with subsequent weeks of VTfb training. Information on these effects is crucial for establishing the most cost-effective use of VTfb training for balance disorders of MS patients.

Training a balance task without VTfb also brings an improvement in trunk sway. However, across a number of studies, we have observed that training alone without VTfb is not as effective as training with VTfb $[35,39,53]$. In this study, we showed that the duration of the carryover period is at least 1 week. Knowing the duration of the carry-over or retention effect is crucial for deciding when the patient needs to return to the treating institution for further training with VTfb. Furthermore, the presence of a strong carry-over effect, or retention of the motor performance, has been cited as a better indicator of motor learning than the improvement registered at the end of a practice session [54]. Based on our experience with 2 training sessions and 2 assessment sessions per week over 2 weeks as used here, it appears that future studies should determine if 4 weeks with 2 training and 2 assessment sessions per week would provide an improved retention of the carry-over effect. 
Figures 3-6 show improvements in balance control with respect to baseline (assessment 1) when assessed with VTfb (assessments 2 and 4). Testing without VTfb (which provided an estimate of the carry-over effect) revealed less significant improvement with respect to baseline (assessments 3 and 5). Thus, an interesting aspect of this study is the rapid improvement in balance control associated with learning to use the VTfb and the retention of the balance skill even when the feedback is no longer present. Little to no research is available on whether these characteristics are typical of skilled motor learning. According to Dayan and Cohen [55], motor skill learning can be divided into fast early phase in which typically significant improvements are achieved with 1 or 2 training sessions (as was the case in our study), and a later slower learning phase in which improvement is seen across multiple practice sessions. Here we noted improvements for the second week of practice sessions which were not significant with respect to those of the first week, possibly because insufficient weeks of practice sessions were given. It has been suggested that the initial fast learning phase is highly dependent on the integrity of the motor cortex [56]. Thus, it would be interesting to compare differences in the initial learning phase of MS patients with and without lesions in the motor cortex and associated brain regions.

The question arises whether we have provided the MS patients we tested with a sensory substitution or a sensory addition using VTfb. Both could be labelled sensory augmentation [34]. As most of the patients suffered motor rather than proprioceptive leg deficits (Table 1), it could be argued that we have provided a sensory addition to help drive appropriate muscle responses correcting unstable balance. We would also argue that because there was a large retention or "carryover" effect that the form of this addition was actuated by reweighting gains of existing vestibular and proprioceptive inputs to balance motor commands. This theoretical framework would explain why we noted no improvement in balance control for non-trained tasks as the test subjects had no VTfb experience with the non-trained tasks. Others have observed similar effects, suggesting that the highly task specific adjustments that occur with sensory augmentation are not observed for tasks which are not trained with sensory augmentation [57].

Previously we have examined the effect of 2 weeks of training with VTfb for older, but healthy, adults and found less improvement compared to a group trained without VTfb [53], in contrast with the current results for which considerable balance improvement in MS patients was achieved with 2 weeks of training. This difference probably depends on the need for sensory augmentation of balance control provided by VTfb of trunk sway and the longer duration of training required for improvement if the need for improved balance control is less. For example, with only one session of VTfb training, chronic bilateral vestibular loss (BVL) patients were shown to reduce sway with improved antagonistic muscle synergies [37]. Thus, the effectiveness of VTfb as a sensory augmentation modality would depend on its necessity for stability. BVL patients cannot continuously stand eyes closed on a foam surface without sensory augmentation [37]. In contrast, healthy older adults who are able to stand under these conditions probably require longer training to improve their balance control. Thus, the period of 2 weeks used by Lim et al. [53] for healthy older adults was probably too short to show a significant effect. Bao et al. [58] used an 8 -week training period and achieved an improvement in dynamic posturography and other balance test batteries as well as a strong carryover effect.

This study has a number of limitations which may have affected our results. Firstly, more significant results might have been obtained, specifically for the carry over effects, if the number of participants had been greater than 15 . In this respect our results must be seen as preliminary. With 15 subjects we obtained more significant results than for our control study [39] with 1 week of VTfb training which had 10 participants. Nonetheless, the current study did not duplicate the control study [39] exactly as the current study had an additional week of VTfb. We assumed that the difference in balance control between training with and without VTfb would be similar in the second week to that in the first week. Another aspect to be taken into account in future studies is that we used a constant set of exercises to train and assess patients on. We specifically excluded difficult exercises such as standing on one leg on a foam surface or walking up and down a set of stairs without handrails as we had noted in previous studies [20] that these tests were too difficult for most MS patients with EDSS scores of 3 and higher to perform. The approach taken by Bao et al. [58] was to adapt the exercise regime to the patient's balance abilities, providing more challenging exercises for those with superior balance control.

\section{Conclusions}

In conclusion, with a 2-week VTfb training program, we were able to significantly improve the balance control of MS patients, even when the VTfb was no longer present. We were not able to improve patients' perceived impressions of their balance control. Future studies will be needed to determine the optimal protocol to establish longer lasting feedback and carry-over effects as well as subjective improvements in balance control.

\section{Conflicts of interest}

John HJ Allum worked as a consultant for the company "Balance International Innovations GmbH" which manufactured the SwayStar ${ }^{\mathrm{TM}}$ posturography system used in this study.

\section{Acknowledgements}

We thank Ms Barbara Wenger for editorial assistance.

\section{Funding}

This research was supported with a grant from the Freie Akademische Gesellschaft Basel to Drs. Ö Yaldizli and JHJ Allum.

\section{References}

1. Pugliatti M, Rosati G, Carton H, Riise T, Drulovic J, et al. (2006) The epidemiology of multiple sclerosis in Europe. Eur J Neurol 13: 700-722. [Crossref]

2. Prosperini L, Castelli L (2018) Spotlight on postural control in patients with multiple sclerosis. Degener Neurol Neuromuscul Dis 8: 25-34. [Crossref]

3. Gunn H, Creanor S, Haas B, Marsden J, Freeman J (2013) Risk factors for falls in multiple sclerosis: an observational study. Mult Scler J 19: 1913-1922. [Crossref]

4. Sosnoff J, Socie M, Boes M, Sandroff B, Pula J, et al. (2011) Mobility, balance and falls in persons with multiple sclerosis. PLoS One 6: e28021. [Crossref]

5. Bethoux F (2013) Gait disorders in multiple sclerosis. Continuum (Minneap Minn) 19: 1007-1022. [Crossref]

6. Kantarci O, Weinshenker B (2005) Natural history of multiple sclerosis. Neurol Clin 23: 17-38. [Crossref]

7. Cameron MH, Horak FB, Herndon RR, Bourdette D (2008) Imbalance in multiple sclerosis: a result of slowed spinal somatosensory conduction. Somatosens Mot Res 25: 113-122. [Crossref]

8. Doty R, MacGillivray M, Talab H, Tourbier I, Reish M, et al. (2018) Balance in multiple sclerosis: relationship to central brain regions. Exp Brain Res 236: 2739-2750. [Crossref]

9. Benedict RHB, Holtzer R, Motl R, Foley F, Kaur S, et al. (2011) Upper and lower extremity motor function and cognitive impairment in multiple sclerosis. $J$ Int Neuropsychol Soc 17: 643-653. [Crossref] 
10. Hamilton F, Rochester L, Paul L, Rafferty D, O'Leary CP, et al. (2009) Walking and talking: an investigation of cognitive-motor dual tasking in multiple sclerosis. Mult Scler J 15: 1215-1227. [Crossref]

11. Hsieh K, Sun R, Sosnoff J (2017) Cognition is associated with gait variability in individuals with multiple sclerosis. J Neural Transm 124: 1503-1508. [Crossref]

12. Kalron A, Aloni R, Dolev M, Frid L, Givon U, et al. (2018) The relationship between gait variability and cognitive functions differs between fallers and non-fallers in MS. $J$ Neural Transm 125: 945-952. [Crossref]

13. Fling BW, Dutta GG, Schlueter H, Cameron MH, Horak FB (2014) Associations between Proprioceptive Neural Pathway Structural Connectivity and Balance in People with Multiple Sclerosis. Front Hum Neurosci 8: 814. [Crossref]

14. Kalron A, Dvir Z, Achiron A (2011) Effect of a cognitive task on postural control in patients with a clinically isolated syndrome suggestive of multiple sclerosis. Eur J Phys Rehabil Med 47: 579-586. [Crossref]

15. Martin CL, Phillips BA, Kilpatrick TJ, Butzkueven H, Tubridy N, et al. (2006) Gait and balance impairment in early multiple sclerosis in the absence of clinical disability. Mult Scler 12: 620-628. [Crossref]

16. Granberg T, Fan Q, Treaba C, Ouellette R, Herranz E, et al. (2017) In vivo characterization of cortical and white matter neuroaxonal pathology in early multiple sclerosis. Brain 140: 2912-2926. [Crossref]

17. Kuhlmann T, Lingfeld G, Bitsch A, Schuchardt J, Brück W (2002) Acute axonal damage in multiple sclerosis is most extensive in early disease stages and decreases over time. Brain 125: 2202-2212. [Crossref]

18. Kirsch V, Keeser D, Hergenroeder T, Erat O, Ertl Wagner B, et al. (2016) Structural and functional connectivity mapping of the vestibular circuitry from human brainstem to cortex. Brain Struct Funct 221:1291-1308. [Crossref]

19. Seemungal B (2014) The cognitive neurology of the vestibular system. Curr Opin Neurol 27: 125-132. [Crossref]

20. Fanchamps MH, Gensicke H, Kuhle J, Kappos L, Allum JH, et al. (2012) Screening for balance disorders in mildly affected multiple sclerosis patients. J Neurol 259: 14131419. [Crossref]

21. Comber L, Sosnoff JJ, Galvin R, Coote S (2018) Postural control deficits in people with Multiple Sclerosis: A systematic review and meta-analysis. Gait \& posture 61: 445-452. [Crossref]

22. Shanahan CJ, Boonstra FMC, Cofré Lizama LE, Strik M, Moffat BA, et al. (2018) Technologies for Advanced Gait and Balance Assessments in People with Multiple Sclerosis. Front Neurol 8: 708. [Crossref]

23. Huisinga J, Yentes J, Filipi M, Stergiou N (2012) Postural control strategy during standing is altered in patients with multiple sclerosis. Neurosci Lett 524: 124-128. [Crossref]

24. Spain RI, St George RJ, Salarian A, Mancini M, Wagner JM, et al. (2012) Body-worn motion sensors detect balance and gait deficits in people with multiple sclerosis who have normal walking speed. Gait \& posture 35: 573-578. [Crossref]

25. Corporaal SH, Gensicke H, Kuhle J, Kappos L, Allum JH, et al. (2013) Balance contro in multiple sclerosis: correlations of trunk sway during stance and gait tests with disease severity. Gait \& posture 37: 55-60. [Crossref]

26. Solomon AJ, Jacobs JV, Lomond KV, Henry SM (2015) Detection of postural sway abnormalities by wireless inertial sensors in minimally disabled patients with multiple sclerosis: a case-control study. J Neuroeng Rehabil 12: 74.

27. Prosperini L, Pozzilli C (2013) The clinical relevance of force platform measures in multiple sclerosis: a review. Mult Scler Int 2013: 756564. [Crossref]

28. Gandolfi M, Geroin C, Picelli A, Munari D, Waldner A, et al. (2014) Robot-assisted vs. sensory integration training in treating gait and balance dysfunctions in patients with multiple sclerosis: a randomized controlled trial. Front Hum Neurosci 8: 318. [Crossref]

29. Cano Porras D, Siemonsma P, Inzelberg R, Zeilig G, Plotnik M (2018) Advantages of virtual reality in the rehabilitation of balance and gait: Systematic review. Neurology 90: 1017-1025. [Crossref]

30. Sienko KH, Balkwill MD, Oddsson LIE, Wall C (2008) Effects of multi-directional vibrotactile feedback on vestibular-deficient postural performance during continuous multi-directional support surface perturbations. J Vestib Res 18: 273-285. [Crossref]

31. Allum JHJ, Carpenter MG, Horslen BC, Davis JR, Honegger F, et al. (2011) Improving impaired balance function: Real-time versus carry-over effects of prosthetic feedback. Conf Proc IEEE Eng Med Biol Soc 2011: 1314-1318. [Crossref]
32. Bark K, Wheeler J, Shull P, Savall J, Cutkosky M (2010) Rotational Skin Stretch Feedback: A Wearable Haptic Display for Motion. IEEE Trans Haptics 3: 166-176. [Crossref]

33. Wall C, Weinberg MS, Schmidt PB, Krebs DE (2001) Balance prosthesis based on micromechanical sensors using vibrotactile feedback of tilt. IEEE Trans Biomed Eng 48: 1153-1161. [Crossref]

34. Sienko KH, Seidler RD, Carender WJ, Goodworth AD, Whitney SL, et al. (2018) Potential Mechanisms of Sensory Augmentation Systems on Human Balance Control. Front Neurol 9: 944. [Crossref]

35. Davis JR, Carpenter MG, Tschanz R, Meyes S, Debrunner D, et al. (2010) Trunk sway reductions in young and older adults using multi-modal biofeedback. Gait \& posture 31: 465-472. [Crossref]

36. Kentala E, Vivas J, Wall C (2003) Reduction of postural sway by use of a vibrotactile balance prosthesis prototype in subjects with vestibular deficits. Ann Otol Rhinol Laryngol 112: 404-409. [Crossref]

37. Honegger F, Hillebrandt IMA, van den Elzen NGA, Tang K-S, Allum JHJ (2013) The effect of prosthetic feedback on the strategies and synergies used by vestibular loss subjects to control stance. J Neuroeng Rehabil 10: 115. [Crossref]

38. Ma CZ-H, Wong DW-C, Lam WK, Wan AH-P, Lee WC-C (2016) Balance Improvemen Effects of Biofeedback Systems with State-of-the-Art Wearable Sensors: A Systematic Review. Sensors 16: 434. [Crossref]

39. van der Logt RP, Findling O, Rust H, Yaldizli O, Allum JHJ (2016) The effect of vibrotactile biofeedback of trunk sway on balance control in multiple sclerosis. Multiple Sclerosis and Related Disorders 8: 58-63.

40. Verhoeff LL, Horlings CGC, Janssen LJF, Bridenbaugh SA, Allum JHJ (2009) Effects of biofeedback on trunk sway during dual tasking in the healthy young and elderly. Gait \& posture 30: 76-81. [Crossref]

41. Polman C, Reingold S, Banwell B, Clanet M, Cohen J, et al. (2011) Diagnostic criteria for multiple sclerosis: 2010 revisions to the McDonald criteria. Ann Neurol 69: 292302. [Crossref]

42. Jacobson GP, Newman CW (1990) The development of the Dizziness Handicap Inventory. Arch Otolaryngol Head Neck Surg 116: 424-427. [Crossref]

43. Hobart JC, Riazi A, Lamping DL, Fitzpatrick R, Thompson AJ (2003) Measuring the impact of MS on walking ability: the 12-Item MS Walking Scale (MSWS-12). Neurology 60: 31-36. [Crossref]

44. Cattaneo D, Regola A, Meotti M (2006) Validity of six balance disorders scales in persons with multiple sclerosis. Disabil Rehabil 28: 789-795. [Crossref]

45. Hegeman J, Shapkova EY, Honegger F, Allum JHJ (2007) Effect of age and height on trunk sway during stance and gait. $J$ Vestib Res 17: 75-87. [Crossref]

46. Allum JH, Adkin AL (2003) Improvements in trunk sway observed for stance and gait tasks during recovery from an acute unilateral peripheral vestibular deficit. Audiol Neurootol 8: 286-302. [Crossref]

47. Goutier KMT, Jansen SL, Horlings CGC, Küng UM, Allum JHJ (2010) The influence of walking speed and gender on trunk sway for the healthy young and older adults. Age Ageing 39: 647-650. [Crossref]

48. Basta D, Rossi-Izquierdo M, Soto-Varela A, Greters ME, Bittar RS, et al. (2011) Efficacy of a vibrotactile neurofeedback training in stance and gait conditions for the treatment of balance deficits: a double-blind, placebo-controlled multicenter study. Otol Neurotol 32: 1492-1499. [Crossref]

49. Kingma H, Felipe L, Gerards M-C, Gerits P, Guinand N, et al. (2019) Vibrotactile feedback improves balance and mobility in patients with severe bilateral vestibular loss. J Neurol 266: 19-26. [Crossref]

50. Nanhoe-Mahabier W, Allum JH, Pasman EP, Overeem S, Bloem BR (2012) The effects of vibrotactile biofeedback training on trunk sway in Parkinson's disease patients. Parkinsonism Relat Disord 18: 1017-1021. [Crossref]

51. Lee A, Bhatt T, Liu X, Wang Y, Pai YC (2018) Can higher training practice dosage with treadmill slip-perturbation necessarily reduce risk of falls following overground slip? Gait \& posture 61: 387-392. [Crossref]

52. Valle M, Casabona A, Cavallaro C, Castorina G, Cioni M (2015) Learning Upright Standing on a Multiaxial Balance Board. PLoS One 10: e0142423. [Crossref]

53. Lim SB, Horslen BC, Davis JR, Allum JH, Carpenter MG (2016) Benefits of multisession balance and gait training with multi-modal biofeedback in healthy older adults. Gait \& posture 47: 10-17. [Crossref] 
Rust HM (2020) Benefits of short-term training with vibrotactile biofeedback of trunk sway on balance control in multiple sclerosis

54. Kantak S, Winstein C (2012) Learning-performance distinction and memory processes for motor skills: a focused review and perspective. Behav Brain Res 228: 219-231. [Crossref]

55. Dayan E, Cohen L (2011) Neuroplasticity subserving motor skill learning. Neuron 72: 443-454. [Crossref]

56. Floyer Lea A, Matthews PM (2005) Distinguishable brain activation networks for short- and long-term motor skill learning. J Neurophysiol 94: 512-518. [Crossref]
57. Giboin L-S, Gruber M, Kramer A (2015) Task-specificity of balance training. Hum Mov Sci 44: 22-31. [Crossref]

58. Bao T, Carender W, Kinnaird C, Barone V, Peethambaran G, et al. (2018) Effects of long-term balance training with vibrotactile sensory augmentation among communitydwelling healthy older adults: a randomized preliminary study. $J$ Neuroeng Rehabil 15: 5. [Crossref]

Copyright: (C2020 Rust HM. This is an open-access article distributed under the terms of the Creative Commons Attribution License, which permits unrestricted use, distribution, and reproduction in any medium, provided the original author and source are credited. 